\title{
Antecedentes de la institucionalización de las organizaciones
}

\section{Antecedents of institutional process}

\author{
Emilio Díez-De-CASTRo ${ }^{1}$ \\ Francisco DíEZ-Martín ${ }^{2}$ \\ Adolfo VÁzQueZ-SÁNCHEZ ${ }^{3}$ \\ Universidad de Sevilla (España) \\ Universidad Rey Juan Carlos (España)
}

Recibido el 16 de mayo de 2013, aceptado el 6 de mayo de 2014

$\mathrm{N}^{\mathrm{o}}$ de clasificación JEL: M14

DOI: $10.5295 / \mathrm{cdg} .130416 \mathrm{ed}$

\section{Resumen:}

Esta investigación es un intento de avanzar en la comprensión de por qué las organizaciones son sensibles a la institucionalización. Para ello, describimos los elementos clave que ayudan a explicar el origen del proceso corporativo de institucionalización. Además, se ha seguido una metodología de investigación cualitativa, utilizando la técnica del 'concept mapping', para agrupar en constructos los diferentes ítems que actúan como factores motivadores de la transformación de las organizaciones en instituciones. Metodológicamente hemos tratado de obviar la separación entre viejo y nuevo institucionalismo siguiendo a los autores que cuestionan la conveniencia de trazar una línea divisoria entre la "vieja" y la "nueva" teoría. Consideramos que el papel del CEO es esencial en el impulso del proceso de institucionalización, aunque en muchas ocasiones sus decisiones estén apoyadas o hayan pasado por el filtro de los equipos de gobierno de la organización o de los consejos de administración. Cualquier impulso que realice la organización dependerá fundamentalmente de las capacidades, las sensaciones, la formación y el modo de pensar del CEO.

Los resultados refuerzan varios de los temas claves sugeridos en la literatura sobre Teoría Institucional. En particular, se establece una clasificación con los motivos que dan origen a las iniciativas institucionales, a saber: autoridad institucional; ventaja en gestión; e, implicación social. Esta clasificación es coincidente, en una gran medida, con los pilares de la institucionalización que han sido definidos en la literatura de la teoría institucional, ayudando a comprender, con mayor detalle, el origen de los procesos institucionales y los antecedentes o motivaciones que los generan y guían.

Palabras clave:

Teoría institucional, institucionalización, iniciativa institucional, motivación gerencial, responsabilidad social de los negocios.

\footnotetext{
${ }^{1}$ Departamento de Administración de Empresas y Marketing de la Universidad de Sevilla. Facultad de Ciencias Económicas y Empresariales, c/ Ramón y Cajal, 1,41018 Sevilla (España). diez@us.es.

${ }^{2}$ Departamento. Economía de la Empresa. Campus de Vicálvaro de la Universidad Rey Juan Carlos, Paseo de los Artilleros, s/n. 28032 Madrid (España).francisco.diez@urjc.es

${ }^{3}$ Departamento de Administración de Empresas y Marketing de la Universidad de Sevilla. Facultad de Ciencias Económicas y Empresariales, c/ Ramón y Cajal, 1,41018 Sevilla (España). adolfov@us.es.
} 


\begin{abstract}
:
This research is an attempt to advance the understanding of why organizations are responsive to the institutionalization process. To this end, we describe key elements that help explain the origin of this process. Furthermore, this research has followed a qualitative research methodology, using the 'concept mapping' technique, and grouping the different constructs items that act as motivating factors in the transformation of organizations to institutions. Methodologically we have tried to overlook the differentiation between old and new institutionalism, this approach follows the ideas of those researchers that question the suitability of drawing a line between the "old" and the "new" theory. We consider that the role of the CEO is essential in driving the institutionalization process, though usually their decisions are supported or have passed through the filter of the organization governance staff or the board of directors. Any progress that the organization makes depends fundamentally on the capabilities, perceptions, training and mindset of the CEO.

The research results reinforce several key topics suggested in the literature on institutional theory. Particularly, we have proposed a classification with the motives that give rise to institutional initiatives: institutional authority; advantage in management; and, social involvement. This classification is consistent, to a larger extend, to the pillars of institutionalization that have been defined in the institutional theory literature, helping to understand, in more detail, the origin of the business processes and the background or motivations that generates and guide them.
\end{abstract}

\title{
Keywords:
}

Institutional theory, institutionalization, institutional initiative, management motivation, corporate social responsibility. 


\section{INTRODUCCIÓN}

La teoría institucional nos indica que cuando las organizaciones se modernizan en su gestión, en su estructura y en su comportamiento, inician un proceso de cambio que las conduce a transformarse en instituciones. Debido a estos procesos de institucionalización que emprenden las organizaciones tradicionales, éstas tienen una mayor probabilidad de sobrevivir y mantenerse en el mercado, además de otras ventajas adicionales (Cruz-Suarez et al. 2014).

El enfoque de nuestro trabajo parte del concepto de institución acuñado en la vieja teoría institucional basado en la aportación de valor a los grupos de interés, un concepto divergente con el de organización tradicional el cual se basa en la preeminencia de los propietarios y de sus objetivos. Aunque en muchos aspectos se aprecia en el presente trabajo que la base metodológica se ha fundamentado en la vieja teoría institucional, en todo momento hemos tratado de obviar la separación entre viejo y nuevo institucionalismo porque compartimos la aportación seminal de Selznick (1996) quien cuestiona la conveniencia de trazar una línea divisoria entre la "vieja" y la "nueva" teoría. La institucionalización es un proceso que se desarrolla en el tiempo y que requiere tiempo para madurar, asentarse y ser interiorizado por la organización. Dicho proceso se inicia cuando aquellos que dirigen la organización toman decisiones, van dando pasos sucesivos, desarrollando iniciativas e integrándolas en la gestión estratégica y la cultura de la organización. Selznick (1957,p. 16) señaló con claridad está cualidad de la institucionalización, "como algo que le sucede a la organización en el tiempo... evolucionan en un proceso amplio y acumulativo".

La institucionalización es un proceso complejo y difícil de implementar, que requiere la puesta en marcha de iniciativas por parte de sus gestores. La adopción de iniciativas institucionales ${ }^{3}$ no ha formado parte de las preocupaciones tradicionales de los directivos de las organizaciones, ya que su actividad generalmente ha sido juzgada por parámetros económicos y financieros. Sin embargo, el cambio en la sociedad que se produjo especialmente en los últimos veinte años del siglo XX y que generó una mayor preocupación por la cuestión social, las expectativas de los trabajadores, el cuidado del medio ambiente y la aceptación de la sostenibilidad, se asentó, en el siglo XXI, dentro de los valores empresariales, favoreciendo los movimientos sobre la responsabilidad social de las empresas y el interés por la mejora de su reputación.

Los especialistas de la teoría institucional se han centrado en buscar explicaciones sobre las instituciones, cómo son, cómo actúan y cómo cambian pero no en cómo surgen, ni qué factores desencadenan los procesos de institucionalización (Scott 1987). En nuestro trabajo queremos avanzar en la explicación de por qué la alta dirección adopta decisiones emprendiendo el camino de la institucionalización. En opinión de Scott (1987), el trabajo de Selznick proporcionó las bases sobre las que asentar la Teoría Institucional y, así, destacar

\footnotetext{
${ }^{3}$ Utilizamos el término iniciativas institucionales, en lugar de iniciativas sociales, iniciativas de responsabilidad social, de sostenibilidad, o iniciativas medioambientales, porque es necesario emplear una expresión que comprenda a todas las demás y, además, recoja algunos aspectos que en otras expresiones quedan al margen de las mismas. Definimos aquí la iniciativa institucional como cualquier programa, práctica, o directriz abordada por una organización, para avanzar en el proceso de institucionalización y conseguir el reconocimiento de su legitimidad.
} 
el importante papel que juegan los directivos en la definición y transmisión de los valores institucionales y desarrollo de las culturas organizativas.

El papel del CEO es decisivo para emprender acciones, tomar decisiones sobre nuevas iniciativas, desarrollar estrategias, reestructurar la organización, etc. Por ello, en el origen de las respuestas institucionales de la organización siempre se encuentra la decisión del CEO, aunque en muchas ocasiones estas decisiones estén apoyadas o hayan pasado por el filtro del equipo de gobierno de la organización. Por tanto, cualquier impulso que realice la organización dependerá fundamentalmente de las capacidades, las sensaciones, la formación y el modo de pensar del CEO (Aguilera et al. 2007).

Comprender los factores desencadenantes del proceso de institucionalización requiere asumir que los CEO son personas que tienen "racionalidad limitada", prejuicios cognitivos, y valores personales, los cuales dirigen sus acciones (Cyert y March 1963; Hambrick y Mason 1984; March y Simon 1958) y que el avance hacia la institucionalización requiere de los directivos una voluntad decidida, una gran conciencia social y responsabilidad ambiental. La literatura nos indica que los CEO, no se apoyan en un solo tipo de motivación, de los estudios se desprende la existencia de un número significativo de motivos como origen de sus iniciativas. Las motivaciones de los CEO son su justificación personal e institucional para tomar iniciativas. También nos indica la literatura que no todos los directivos se basan en las mismas motivaciones. El resultado es que si las motivaciones de los directivos son diferentes, también lo serán las iniciativas que se adopten como consecuencia de dichas motivaciones. Los resultados conducen a que los procesos de institucionalización serán distintos entre las organizaciones, desarrollándose en cada caso peculiaridades que las identifican y diferencian.

La cuestión es relevante por varios motivos, en primer lugar, porque el papel de las instituciones ha ido creciendo con el tiempo y posiblemente seguirá aumentando (Greenwood y Suddaby 2006) en segundo lugar, porque la institucionalización de las organizaciones representa el progreso en la gestión de las mismas. Este papel relevante de las instituciones no se menciona únicamente por los estudiosos de la teoría institucional, otros enfoques teóricos, como el de los costes de transacción, también han aludido a la importancia del papel de las instituciones (Roberts y Greenwood 1997; Williamson, 1991; Williamson, 2000).

La identificación de las motivaciones o fuerzas desencadenantes de las iniciativas institucionales, se ha realizado a partir de la revisión de la literatura científica dentro del campo de las iniciativas institucionales. Siguiendo dicha literatura, se ha elaborado una relación descriptiva de variables desencadenantes de las iniciativas institucionales, permitiendo establecer una tipología de las mismas. Dicha tipología se realizó utilizando mapas conceptuales como herramienta de análisis.

\section{DESENCADENANTES DE LA RESPUESTA INSTITUCIONAL}

La cuestión de los factores desencadenantes de las iniciativas institucionales, en los años 70 del siglo pasado ya era considerada relevante para entender los procesos de institucionalización. Las respuestas de los investigadores a estas cuestiones acostumbran a ser demasiado generales para que puedan explicar de una forma clara y contundente el modo de actuar de los directivos superiores de la organización. 
Sin fuertes motivos, los directivos difícilmente adoptarán decisiones de contenido institucional, pues necesitan demostrar que sus decisiones son racionales y que éstas se deben a criterios y argumentaciones que apoyan y avalan las actuaciones emprendidas. Cuando los actores emprenden actividades, lo hacen a partir de un comportamiento racional. La racionalidad determina que "los actores en las empresas busquen obtener ganancias; que los actores en los partidos políticos busquen conseguir votos; y, que los actores en los centros de investigación persigan publicar sus hallazgos" (Scott 1987, p. 508). Sin embargo, estas decisiones no son completamente racionales, los líderes de las organizaciones siempre se han movido por una combinación de comportamientos en los que se mezclan sensaciones con creencias y análisis económico racional.

En general, las empresas menos institucionalizadas, de nueva creación o de menor dimensión, son las que presentan una mayor carga de actuaciones directivas derivadas de sensaciones y experiencias y, en menor lugar, de análisis racional de las mismas (Hatchuel 2001). Por el contrario, las organizaciones con mayor nivel de institucionalización son las que en mayor medida toman sus decisiones apoyándose en aspectos racionales que han sido analizados e informados previamente, y al amparo de órganos de decisión colectivos para dar mayor seguridad y coherencia a las mismas.

En consecuencia, es complejo determinar si las iniciativas institucionales están guiadas por valores morales o si son impulsadas por preocupaciones de carácter estratégico, como la protección de los beneficios de la empresa o la conservación de la legitimidad de la organización (Graafland y Van der Ven 2006; Díez-Martín et alia 2013). Las fuerzas que desencadenan estos procesos tienen un origen distinto, pero en la literatura se han tratado con frecuencia aunque de forma individualizada. Del análisis de las aportaciones de los estudiosos hemos podido identificar un total de 16 factores desencadenantes de las iniciativas de institucionalización de los CEOs, que pasamos a comentar.

\subsection{Regulación coercitiva}

Los gobiernos tienen distintas formas de influir sobre la actividad de las organizaciones pero la que se ha considerado habitualmente como más potente es la que se realiza a través de la legislación ejerciendo presiones coercitivas. El poder coactivo del gobierno se ejerce a través de reglas administrativas, órdenes judiciales y normas legales (Maguire y Hardy 2009; Pache y Santos 2010) pero además vigilan el cumplimiento y sancionan los comportamientos de las organizaciones que se separan de lo legislado (Heugens y Lander 2009). La legislación se utiliza como un instrumento para reafirmar la necesidad del cumplimiento de las normas de un modo coactivo (DiMaggio y Powell 1983) ayudando a evitar comportamientos irresponsables de la organización (Campbell 2007). La conformidad con las normas legales, se produce en respuesta a las presiones coercitivas y es, a menudo, el resultado de que los actores tratan de evitar las penas asociadas con el incumplimiento (Maguire y Hardy 2009).

La legislación es un mecanismo gubernamental de presión sobre las organizaciones que se origina para evitar un comportamiento corporativo irresponsable, sobre la base de que las empresas operan de manera oportunista si creen que pueden actuar del modo en que deseen (Campbell 2007). 
Las presiones institucionales motivan a las organizaciones para incrementar su legitimidad con respecto a los componentes institucionales (Dacin et al. 2007; Scott 1995). Los estudios empíricos han demostrado que las organizaciones ganan legitimidad mediante la conformidad con las normas y reglamentos impuestos por autoridades externas (Deephouse 1996; Ruef y Scott 1998; Bitektine 2011). De ahí, que un factor desencadenante de iniciativas institucionales es la actitud favorable del CEO para el cumplimiento de las normas obligatorias que emanan de los gobiernos.

\subsection{Autorización gubernamental}

La legislación no siempre es imperativa, en muchas ocasiones no obliga al cumplimiento de normas específicas mediante la coacción. Los gobiernos pueden utilizar otros mecanismos para conseguir un comportamiento de las organizaciones basado en sus objetivos y prioridades públicas, tales como la autorización, la incentivación y la señalización (Scott 1987).

La autorización consiste en "la legitimación por una entidad superior de las características o cualidades de una organización para desarrollar una actividad" (Scott 1987, p. 502). La naturaleza de la autorización puede ser pública o privada (Campbell 2007) pues a veces las normas no proceden de la administración pública, sino de asociaciones profesionales, gremiales o de otro tipo, a quienes se confiere la autoridad institucional de algún tipo. La presencia de organizaciones independientes no gubernamentales y otras que evalúan el comportamiento empresarial, las normas institucionalizadas sobre el comportamiento corporativo apropiado, el comportamiento entre las organizaciones y sus grupos de interés (Campbell 2007) son la base de la aparición de normas vinculadas a la autorización. En ocasiones, para participar en determinados proyectos y contratos, las organizaciones deben disponer de una acreditación previa demostrando el cumplimiento de normas o estándares establecidos. En estos casos, el cumplimiento y la observancia de las normas, aunque inicialmente no tienen un carácter obligatorio (Dornbusch y Scott 1975), goza de una condición similar a la impuesta por las autoridades públicas. La falta de autorización puede llevar a la expulsión de los miembros asociados que no cumplan con las mismas.

Las organizaciones se someten a la autorización como un factor que incide directamente en su legitimación (Vaara y Tienari 2008). La autorización se plasma en ocasiones en alcanzar la certificación. Sine et al. (2007) consideran que la certificación es una de las pocas señales creíbles de legitimidad que una organización puede proporcionar cuando la legitimidad del sector es baja. Para que la autorización tenga éxito, las organizaciones deben compartir un consenso acerca de los comportamientos esperados, y además, los comportamientos deben ser observables y los procesos de aplicación tienen que ser coherentes (Ostrom 2000). La frecuencia con que encontramos estos procesos de autorización, en una amplia variedad de sectores, sugiere que en muchas organizaciones las ganancias asociadas superan ampliamente los costes asociados a la autorización.

\subsection{Incentivación de actuaciones}

En ocasiones los gobiernos pueden estar en posición de ofrecer fuertes incentivos a las organizaciones que actúen conforme a sus deseos (Maguire y Hardy 2009). Las insti- 
tuciones pueden atraer a los actores para comportarse de cierta manera a través del uso de incentivos positivos, recompensas y otros mecanismos (Campbell 2007).

Las estrategias de incentivación crean cambios estructurales y organizativos en quienes están dispuestas a ajustarse a las condiciones del gobierno. Normalmente, el gobierno especifica las condiciones para acceder y permanecer entre los que tienen acceso a la financiación o recompensa por determinados trabajos realizados. Las estrategias de incentivación, al ser seguidas por un importante número de organizaciones que se adaptan a las pautas o realizan las actividades marcadas por las condiciones de los incentivos (Matten y Moon 2008), crean un mayor isomorfismo o similitud estructural en un sector económico. El motivo más importante para aceptar la incentivación es captar los recursos que permiten la inclusión de la organización en procesos apoyados por los gobiernos, lo cual, en muchos casos, contribuyen a su modernización.

Los incentivos externos pueden provocar que las organizaciones busquen la adhesión a normas, incluso si las normas no son internalizadas (Ostrom 2000). Uno de los motivos que originan el fracaso de los objetivos originales de los procesos de incentivación surge por la falta de incentivos intrínsecos en la organización, lo que impide la interiorización de normas (Terlaak 2007).

El rechazo de participar en el sistema de incentivación puede ser sancionado por el cese de ciertas relaciones sociales, el ostracismo de la organización y la reducción de futuros intercambios económicos (Ingram y Silverman 2002).

\subsection{Señalización de políticas públicas}

Los gobiernos, a través de diversos mecanismos de comunicación, transmiten a las organizaciones cuáles son sus deseos y aspiraciones respecto a los comportamientos y actitudes de las mismas (Spence 1973).

Las organizaciones saben que su diálogo con los gobiernos está parcialmente condicionado por su capacidad de sincronizar o armonizar sus actuaciones con las políticas públicas o los enfoques políticos de los gobiernos. Para las organizaciones, seguir las señales e indicadores que emanan de los gobiernos, es un requisito no formal para mantener unas relaciones fluidas con ellos, y la ausencia de dicha sincronización acostumbra a generar barreras en dichas relaciones. Por otro lado, también existe la conciencia en las organizaciones que si no emprenden iniciativas institucionales, los legisladores pueden obligarles a ello (Brønn y Vidaver-Cohen 2009).

Terlaak (2007), señala que las normas, reglas informales y códigos de comportamiento, pueden crear 'orden sin ley', completando las leyes y reglamentos con las iniciativas no obligatorias, como la autorización, la incentivación y la señalización.

\subsection{Acceso a recursos}

Algunos autores señalan que las organizaciones deben crear una impresión de viabilidad y legitimidad antes de poder recibir cualquier apoyo (Starr y MacMillan1990; Dacin et al. 2007). De este modo, existe el convencimiento de que una organización institucionalizada puede conseguir clientes más fácilmente, entablar relaciones con proveedores y, en definitiva, incrementar el acceso a recursos críticos para alcanzar el éxito (Baum y Oliver 
1991). Al adoptar estructuras y estrategias que son vistas como racionales y apropiadas, las organizaciones consiguen acceso a los recursos más atractivos en condiciones más favorables (Heugens y Lander 2009).

Cuando una organización se encuentra institucionalizada, está indicando que es apropiado invertir en ella pues, a pesar de la incertidumbre sobre el futuro de los resultados financieros, la institucionalización mejora la estabilidad y comprensibilidad de las actividades organizativas (Singh et al. 1986), mientras que la falta de legitimidad estaría asociada a una menor capacidad de acceso a los recursos (Zimmerman y Zeitz 2002).

\subsection{Interés estratégico}

Los directivos de todo el mundo perciben la participación en iniciativas sociales como algo muy beneficioso para la estrategia del negocio (Davis 1973). Brønn y Vivader-Cohen (2009) encontraron que las iniciativas sociales podían mejorar la rentabilidad, fortalecer la reputación, y fomentar el compromiso de los trabajadores con la empresa. Tudway y Pascal (2006) afirmaban que el beneficio para los accionistas puede maximizarse cuando las empresas cumplen con una agenda social visible. De ahí, que en muchas ocasiones exista una actitud positiva del CEO para emprender actuaciones insitucionales con el objeto de conseguir desarrollar su estrategia y alcanzar ventajas competitivas, incluso algunos investigadores (Cho y Patten 2007) apoyan la idea de que los motivos estratégicos han sustituido a los altruistas para determinar la implicación social de una organización.

La evaluación de los resultados en el largo plazo es otro de los efectos positivos que pueden motivar a las organizaciones a realizar actuaciones socialmente responsables. "la sociedad espera de las empresas que cumplan con una serie de beneficios sociales, y deben producir esos beneficios si esperan obtener beneficios a largo plazo" (Davis 1973, p. 321).

Varios estudios han demostrado (Gardberg y Fombrun 2006; Glaskiewicz y Colman 2006; Dacin et al. 2007) que las organizaciones desarrollan iniciativas institucionales porque los gerentes creen que estas actividades pueden generar una ventaja económica, proporcionar oportunidades de nuevos negocios, evitar la regulación, o ayudar a la compañía a cumplir con las demandas de los accionistas.

\subsection{Fuertes relaciones con el sector público}

Un factor clave es la disposición del CEO para operar ajustándose a las expectativas gubernamentales, especialmente cuando su organización tiene sus ventas o recursos fuertemente comprometidos con el sector público.

Además, fortalecer las relaciones entre la empresa y el sector público puede ser fundamental para crear nuevo conocimiento dentro de las organizaciones, tanto públicas como privadas (Drucker 1999). Dado que las organizaciones públicas operan en un entorno en el que hay muchas restricciones formales, habitualmente deben encontrar nuevos métodos de gestión para sacar adelante su misión con éxito. Esas habilidades desarrolladas por el sector público pueden ser objeto de estudio y adopción por las empresas privadas. 


\subsection{Innovación y mejora de procesos}

A la hora de identificar los motivos que conducen a la institucionalización de las organizaciones, una cuestión que no ha sido suficientemente tratada ha sido la importancia de la innovación. Para Hart (1995) las oportunidades económicas surgen por motivaciones ligadas a la mejora de los procesos de producción, el aprendizaje y la mejora de capacidades.

Por ejemplo, las organizaciones que han intentado adaptarse a las normas y estándares de la sociedad mediante actuaciones medioambientales, les ha llevado a mejoras en los sistemas y la tecnología empleada (Bansal y Roth 2000). El reciclado de los desechos ha llevado a aplicar nuevas tecnologías, nuevas formas de organización de la producción, métodos diferentes de almacenamiento y sistemas novedosos de eliminación para evitar la contaminación. También se ha comprobado que los ingresos pueden mejorarse a través de la comercialización de productos ecológicos e incluso de la gestión de los residuos como material para ser reciclado (Shrivastava 1995).

\subsection{Actitud del consumidor}

Sen y Bhattacharya (2001) demuestran la existencia de relación entre las iniciativas sociales de las empresas y las decisiones de compra de los consumidores. Los consumidores ejercer su influencia a través de su poder de compra, o su disposición a pagar más por ciertos bienes y servicios. Pero también de forma colectiva, por medio de movimientos sociales, por ejemplo, mediante el boicot a: los alimentos transgénicos y las campañas de los cultivos en la UE, a la empresa Nike en la década de 1990 (Kozinets y Handelman 2004). Cada vez existe una mayor conciencia de la relación positiva entre las acciones institucionales de la empresa y la reacción de los consumidores, así como a los efectos negativos cuando los comportamientos institucionales son perjudiciales, o percibidos como no legítimos (Sen y Bhattacharya 2001).

\subsection{Reducción de problemas y costes}

Davis (1973, p. 317) mantiene que "si la empresa remolonea y deja de afrontar hoy los problemas sociales, puede que en el futuro se vea ocupada constantemente en apagar fuegos en el ámbito social y deje de tener tiempo para dedicarlo a su objetivo fundamental: producir bienes y servicios. Dado que es inevitable abordar estos problemas sociales más tarde o más temprano, es en realidad más económico afrontarlos ahora, antes de que crezcan para convertirse en importantes batallas que consuman la mayoría del tiempo de los directivos". Numerosos estudios avalan estas afirmaciones.

Dacin et al. (2007) afirmaba que las organizaciones cuyas actuaciones van en contra de su legitimidad pueden encontrarse: que se les niegue el acceso a mercados decisivos, no encontrar socios para llevar a cabo proyectos conjuntos y compartir los riesgos, la exclusión de la financiación gubernamental y el rechazo de los clientes, poniendo así en peligro el potencial de su ventaja en gestión.

Green et al. (2009) afirman que la literatura TQM (Total Quality Management) teoriza la noción de que la calidad se puede mejorar sin aumentar los costes, debido a las prácticas de buena gestión, por ejemplo, reduciendo los residuos (Easton y Jarrell 1998; Porter y Van 
der Linde 1995). Según esta lógica, la mejora de la calidad debería aumentar las ventas de los productos, y la reducción del coste de los productos (Green et al. 2009).

Bansal y Roth (2000) argumentan que las decisiones de los gestores tienen como objetivo fundamentalmente reducir los costes y evitar los riesgos económicos del incumplimiento de la normativa vigente. En esta línea, es importante conocer que las acciones ilegales y poco éticas tienen enormes costes para la sociedad y para las propias organizaciones (Pfarrer et al. 2008).

\subsection{Integración en redes sociales}

La participación en redes sociales crea convergencia de actitudes y comportamientos. Aquellos que realizan actividades sociales tienden a conectar con organizaciones similares. Fomentando relaciones que pueden llegar a ser muy estables por la mutua confianza que proporciona participar en valores comunes. De ahí, la formación de redes sociales como una forma natural de actuación que facilita el contacto previo con otros mercados, y la accesibilidad e integración en redes mundiales que favorecen el crecimiento de la organización.

\subsection{Presiones de los grupos de interés}

La teoría de los grupos de interés (Freeman 1984) da cuenta de la diversidad de intereses de los actores y de cómo ejercen una gran influencia sobre los CEO, a la hora de la toma de decisiones (Díez-Martín et al. 2008). Los gestores justifican la adopción de iniciativas institucionales cuando existen presiones externas. De este modo, evitan sanciones sociales (protestas, informaciones negativas en prensa, o el deterioro de la imagen) que puedan dañar sus relaciones con los accionistas. Está probado que los clientes, comunidades locales, grupos de defensa del medio ambiente, e incluso el medio ambiente natural en sí, animan a las empresas a considerar los impactos ecológicos en su toma de decisiones (Riquel y Vargas 2013). De este modo, los responsables de las organizaciones actuarán garantizando el bienestar de los diferentes grupos que participan o se relacionan con la empresa.

\subsection{Mimetismo}

Para las personas, mantener comportamientos diferentes a los que se producen en su marco social acostumbra a tener repercusiones tales como: la exclusión del grupo, la marginación e incluso el rechazo. Los nuevos institucionalistas descubrieron que las organizaciones sufrían un efecto similar al de las personas. Así, para evitar los efectos negativos de la singularidad, adoptaban comportamientos similares a los de los líderes del sector. El mantenimiento de actuaciones similares o miméticas con otras organizaciones del sector se traduce, en la práctica, en hacer lo que hacen los demás y, en función de ello, conseguir la legitimidad que proporciona ser uno más del grupo (Suchman 1995).

DiMaggio y Powell (1983) ponen de manifiesto que ante situaciones de incertidumbre, la decisión más eficiente no es siempre conocida a priori. En estas situaciones, mientras mayor sea la incertidumbre sobre la eficiencia de las alternativas, menor impacto tendrán 
las características de eficiencia de las alternativas y mayor será el impacto que tendrán las fuerzas institucionales miméticas sobre la toma de decisión (Palmer et al, 2008). Por ejemplo, Peloza (2003) encuentra que, cuando ciertas empresas de referencia "suben el listón" a la hora de involucrarse en iniciativas sociales, las demás sienten la necesidad de aumentar su participación en iniciativas sociales para igualarse a sus competidores.

De este modo, con el paso del tiempo, las organizaciones tienden a parecerse unas a otras, incluso cuando esta tendencia no conduce a mejorar la eficiencia organizativa. Existe consenso entre los institucionalistas sobre la bondad del isomorfismo para las organizaciones, ya que "evita la confusión, las hace comprensibles, las convierte en legítimas, les facilita la financiación y evita sanciones coercitivas del Estado" (Heugens y Lander, 2009, p. 63).

\subsection{Prestigio social}

La satisfacción de haber creado una institución reconocida, la obra transcendente que representa la existencia de una institución, es clave para entender el prestigio social como factor motivador. La marca de coches Ferrari puede tener una gran imagen como constructor pero lo que le proporciona seguidores, personas involucradas y orgullosas de ser partidarias de sus coches, lo que provoca el mito de la institución, es su prestigio social. Un ejemplo, la declaración de Steve Jobs "Mi objetivo ha sido siempre, no sólo construir grandes productos, sino también levantar grandes empresas" (Isaacson 2011, p. 556). La simbiosis entre prestigio individual del CEO y prestigio social de la organización es un factor que lleva a desarrollar actividades institucionales.

Sin duda, el prestigio de la organización crece cuando actúa de la forma en que espera o desea la sociedad. Actuar de acuerdo con las expectativas de la sociedad puede mejorar la reputación de la organización, favoreciendo la consecución de nuevos recursos o la obtención de facilidades por parte de los gobiernos para su desarrollo. Se ha demostrado que las iniciativas sociales fortalecen notablemente la reputación corporativa y ayudan a reducir el riesgo (Hart 1995; Russo y Fouts 1997). Así, las inversiones en iniciativas sociales pueden ser tan importantes para la rentabilidad como las inversiones en publicidad o en I+D (Gardberg y Fombrun 2006).

Greenwood y Suddaby (2006) señalan que cuando algunos miembros de una industria inciden en conductas reprobables o cuando el conjunto de la industria es percibida como poco sensible a las necesidades sociales, se ha comprobado que, basándose en la existencia de una "reputación de los comunes", todas las empresas de la misma industria se ven "salpicadas por el mismo lodo". En estas industrias, los directivos actúan desplegando esfuerzos y recursos adicionales para transmitir la imagen de que sus organizaciones son "socialmente proactivas", y se diferencian de sus colegas menos responsables (Barnett 2007). 2.15. Posición ética y valores corporativos

Carroll (1979) hace referencia a las responsabilidades éticas de las corporaciones. Las conductas apropiadas por las que deberían regirse las organizaciones se deben basar en las normas éticas de la sociedad (Meyer y Rowan 1983) y con ello las organizaciones ganan legitimidad (Suchman 1995; Deephouse 1996). Las empresas éticamente motivadas actúan con comportamientos socialmente responsables porque es lo correcto, se han documentado actuaciones en las que "hacer lo correcto", parece un motivo más fuerte para la iniciativa 
social que los beneficios que se puedan generar para la organización (Hahn y Scheermesser 2006; Steurer et al. 2005).

El CEO y los miembros del equipo de dirección son fundamentales para alentar a las organizaciones a comportarse de un modo que realce su papel ético en la sociedad. No obstante, es prácticamente indistinguible la posición personal de los directivos superiores y sus valores, con aquellos otros que tiene interiorizado la organización, existe una simbiosis entre valores del CEO y los corporativos.

Bartlett y Preston (2000) se preguntaban: ¿Puede el comportamiento ético existir realmente en un negocio? La Teoría de la Administración sugiere que los actores organizacionales conducen a las organizaciones basándose en la moral, o los valores de la empresa, lo que la lleva más allá de los intereses económicos (Davis, 1973). Los actores organizacionales dentro de las empresas, tales como los equipos de dirección, toman decisiones en función de sus sesgos cognitivos y valores personales (Cyert y March 1963), los cuales se difunden a través de los valores generales de la organización y la ética empresarial generando la obligación moral de "hacer lo correcto".

\subsection{Filantropía}

Surge por la creencia del CEO que la organización debe compensar a la sociedad en general y ayudar a grupos de interés no relevantes o completamente ajenos a su actividad.

La filantropía es una de las formas que tienen las organizaciones de corresponder o devolver a la sociedad parte de lo recibido de ellas. La filantropía significa que las iniciativas se emprenden por responsabilidad con la sociedad y el género humano, no por interés. Son considerados actos filantrópicos: las donaciones a fundaciones con fines humanitarios, el apoyo a instituciones que necesitan ayuda para mejorar la calidad de la vida humana en su esfera de actividad, el trabajo voluntario, las aportaciones ante desastres humanitarios, etc. Carroll (1979) considera que las actividades filantrópicas son medidas discrecionales adoptadas por las entidades empresariales que están más allá de sus obligaciones económicas, jurídicas y éticas. Las actuaciones filantrópicas proporcionan fondos $\mathrm{u}$ otras ayudas a grupos que no son relevantes o que no tienen capacidad de influencia e incluso, a grupos que ni siquiera pueden catalogarse como grupos de interés.

Ashforth y Gibbs (1990), argumentan que la filantropía corporativa puede utilizarse como una herramienta de legitimación, compartiendo recursos con organizaciones sin ánimo de lucro, esponsorizando iniciativas sociales en países menos desarrollados, o adoptando actuaciones proactivas para proteger el patrimonio natural (Williams y Barrett 2000; Chen et al. 2008; Rehbein et al. 2004; Matten y Moon 2008).

\section{METODOLOGÍA}

Para definir los constructos básicos que forman las fuerzas desencadenantes de las iniciativas institucionales utilizamos la metodología de los mapas conceptuales, un método ampliamente utilizado para especificar los marcos conceptuales objeto de estudio (Kane y Trochim 2007; Trochim 1989). Los mapas conceptuales han sido aplicados de diferentes maneras (Rosas, 2005), contribuyendo al desarrollo de modelos lógicos (Anderson et al. 2006; Yampolskaya, et al. 2004) y el establecimiento de criterios (Barth 2004). Además, 
en la literatura de evaluación se ha resaltado la importancia de la integración de las perspectivas de los actores mediante su participación en los procesos de construcción de mapas (Fine et al.2000; Huebner 2000).

La identificación previa de 16 variables crea una alta complejidad para el análisis y no permite visualizar una estructura conceptual clara para definir y hacer operativos los factores desencadenantes de la respuesta organizativa, lo que conduce a la redundancia de esfuerzos y la sobrecarga de información. La técnica de los mapas conceptuales ayuda a resolver esta cuestión. Esta técnica fue elegida por varios motivos: a) No se basa en esquemas de codificaciones preconcebidas; b) Funciona por encima del nivel de análisis sintáctico; c) Conserva el contexto del concepto en la unidad de análisis; d) Va más allá de contar la repetición de palabras, representando la coocurrencia de juicios de similitud.

El número óptimo de participantes para la elaboración de la clasificación y agrupamiento de los ítems no ha sido determinado de una forma precisa, ni por los creadores de la herramienta ni por aquellos investigadores que la han utilizado. Sin embargo, existen recomendaciones al respecto que hemos de tener en cuenta. En primer lugar, Trochim (1993) sugiere que el número mínimo de participantes se puede situar en 10 (Jackson y Trochim 2002). Por otra parte, en el mismo estudio, Trochim analizando un número importante de investigaciones, llegó a la conclusión que la media de participantes se encontraba próximo a 15.

En cuanto a la selección de los participantes se deben seguir criterios de diversidad y calidad de los mismos. A tal efecto reclutamos 12 participantes, todos ellos con estudios universitarios superiores que desarrollan su actividad en España. A tal efecto podemos señalar que cinco de ellos son doctores, cuatro son licenciados y tres son magister. Este grupo desarrolla su actividad tanto en el sector público como en el privado. Cinco de ellos son profesores de tres universidades diferentes, tres de los cuales tienen experiencia en la gestión de empresas públicas y dos en la gestión de empresas privadas. Además, el grupo se completó con siete personas más, uno de ellos es directivo de empresa multinacional, dos son empresarios, uno de empresa multinacional y otro de empresa nacional. Incorporamos dos consultores, uno de gran empresa y otro de empresa pública. Por último, en el grupo se integró el presidente de una fundación y un directivo de PYME.

El cuestionario se entregó en papel, acompañado de un juego de tarjetas con los nombres de los ítems para facilitar la clasificación y unas líneas explicando brevemente el contenido de los mismos. Se comentó a los participantes el significado de las variables, bien personalmente o por vía telefónica, con objeto de que no se generaran dudas sobre su interpretación. En el cuestionario se incluyeron todos los ítems (16) que previamente se habían identificado en la categorización desarrollada en base a la literatura. A los doce participantes se les facilitaron unas instrucciones para agrupar los 16 ítems en pilas, para ello debían decidir individualmente cuáles ítems en su opinión tienen una mayor relación entre sí. Se pusieron cuatro restricciones, las cuales son habituales en la utilización de esta técnica (Rosas y Camphausen 2007): a) todos los ítems no pueden colocarse en una misma pila; b) todos los ítems no podían separarse en pilas distintas; c) los ítems no podían figurar a la vez en dos pilas diferentes; d) los ítems no pueden colocarse en una pila de varios o miscelánea.

Usamos el Concept System desarrollado por el Cornell Office for Research on Evaluation (CORE) pero no su software estadístico pues decidimos utilizar el SPSS. El instru- 
mento desarrollado por el Concept System como input para el análisis de conglomerados jerárquicos, usa los datos en dos dimensiones de la configuración derivada a partir del análisis de escalamiento multidimensional.

El análisis cluster comprende una serie de métodos multivariados que se utilizan para encontrar verdaderos grupos de ítems. El método de análisis que se utiliza en este estudio es de agrupamiento jerárquico, el cual tiene la ventaja de no exigir el conocimiento a priori del número de grupos. Una revisión realizada por Sharma (1996) sugiere que el procedimiento de agrupación de Ward puede ser el mejor, por lo que decidimos que fuera el utilizado en este estudio. Como medida de distancia, se empleó la distancia euclidea al cuadrado, que es una de las medidas más comúnmente adoptadas.

\section{RESULTADOS}

El mapa, comenzó con la construcción de una matriz de similitudes simétrica, Xij, de 16 x 16. Para dos elementos i y j, se pone un 1 en Xij si los dos elementos se colocan en el mismo grupo por el participante, en caso contrario, se introduce un 0 . Respecto a la matriz de similitud total de 16 x 16, Tij se obtiene sumando las matrices individuales de Xij. Por lo tanto, cualquier celda en esta matriz podría tomar valores enteros entre $0 \mathrm{y}$ el número de personas que ordenan las variables. La matriz de similitud total fue analizada mediante análisis de escalamiento multidimensional (MDS) con una solución de dos dimensiones (Kruskal y Wish, 1978), cuyo resultado se recoge en el Gráfico 1.

Grafico 1

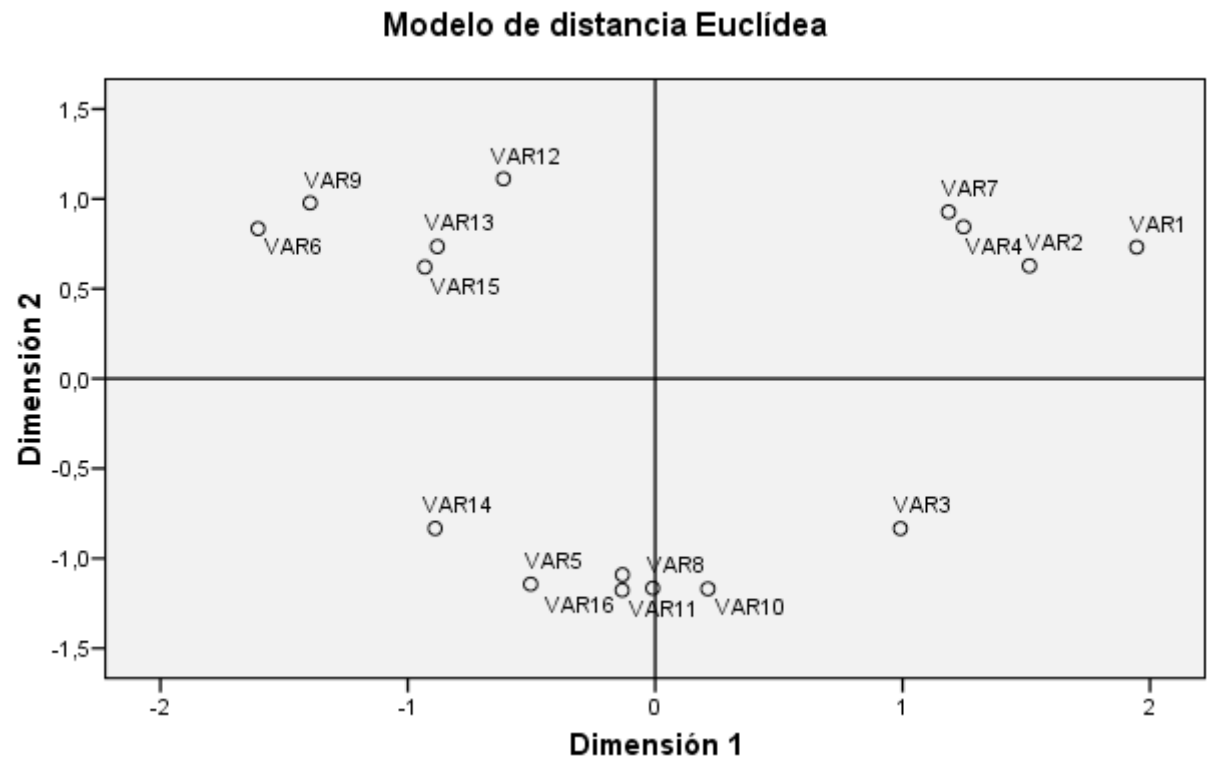


La bondad de ajuste del mapa a la matriz original se midió mediante el estadístico stress calculado mediante la fórmula de Kruskal. El stress es una medida de la bondad del ajuste para la solución del escalamiento multidimensional. La distancia entre los puntos refleja el grado de similitud entre ellos, unas distancias más pequeñas corresponden a más similitud y las distancias más grandes corresponden a menos similitud (Yampolskaya et al. 2004). Trochim (1993) comprueba que los estudios que utilizaban mapas conceptuales los valores de stress se encontraban entre un mínimo de 0.15526 y un máximo de 0.35201 . Trochim, reconoce que la literatura sobre escalamiento multidimensional sugiere niveles de stress más bajos que los que se obtienen típicamente en los mapas conceptuales, sin embargo, señala que las recomendaciones de la literatura se basan típicamente sobre experiencias de fenómenos mucho más estables y precisos que los abordados en el campo social. En nuestro análisis el valor de stress fue 0.339 cercano al nivel superior de la horquilla, y la proporción de la varianza de las diferencias de los datos fue RSQ $=0.622$.

Aunque en este caso el gráfico nos da una aproximación visual, es necesaria la confirmación de las agrupaciones mediante el análisis cluster. El análisis de conglomerados jerárquicos de las coordenadas MDS, permiten, como resultado, generar los mapas que muestran las variables individuales en un espacio bidimensional $(\mathrm{x}, \mathrm{y})$ con variables similares situadas más cerca entre sí, mostrando cómo se agrupan las variables. La naturaleza de ramificación del dendograma obtenido como resultado permite identificar la pertenencia a un grupo rastreando hacia atrás o hacia adelante para cualquier caso individual o de grupo. Además, da una idea de cuán grande es la distancia entre los casos que se agrupan utilizando una escala de 0 a 25 a lo largo de la parte superior de la tabla. Cuanto más grande sea la distancia antes de que se unan dos grupos, más grandes serán las diferencias entre estos grupos. Para encontrar un número de ítems de un grupo en particular, basta con rastrear hacia atrás a través de la escala. En todo los casos podemos considerar que un grupo que se encuentra en la escala por debajo de 5 es aceptable para el análisis.

Los resultados indican la existencia de tres clusters que incluyen los 16 desencadenantes de las iniciativas institucionales (Tabla 1 y ver Apéndice).

Tabla 1

\section{Conglomerado de pertenencia}

\begin{tabular}{|c|c|c|c|c|c|}
\hline Item & Contenido & Cluster & Item & Contenido & Cluster \\
\hline 1 & Regulación coercitiva. & 1 & 9 & Actitud del consumidor. & 3 \\
\hline 2 & Autorización normativa. & 1 & 10 & $\begin{array}{l}\text { Reducción de problemas y } \\
\text { costes. }\end{array}$ & 2 \\
\hline 3 & Incentivación de actuaciones. & 2 & 11 & $\begin{array}{l}\text { Integración en redes } \\
\text { sociales. }\end{array}$ & 2 \\
\hline 4 & $\begin{array}{c}\text { Señalización de políticas } \\
\text { públicas. }\end{array}$ & 1 & 12 & Presión de los stakeholders. & 3 \\
\hline 5 & Acceso a recursos. & 2 & 13 & Mimetismo. & 3 \\
\hline 6 & Interés estratégico. & 3 & 14 & Prestigio social. & 2 \\
\hline 7 & $\begin{array}{l}\text { Fuertes relaciones con el sector } \\
\text { público. }\end{array}$ & 1 & 15 & $\begin{array}{c}\text { Posición ética y valores } \\
\text { corporativos. }\end{array}$ & 3 \\
\hline 8 & $\begin{array}{l}\text { Innovación y mejora de proce- } \\
\text { sos. }\end{array}$ & 2 & 16 & Filantropía. & 2 \\
\hline
\end{tabular}


Los clusters sugieren que existen tres motivaciones básicas para emprender iniciativas institucionales, a las que hemos denominado: Autoridad institucional; Ventaja en gestión; e, Implicación social.

Estos constructos nos acercan a conceptos tradicionales en teoría institucional como los pilares de las instituciones definidos por Scott (1995), y también guardan afinidad con los encontrados en los trabajos de otros investigadores como Brønn y Vidaver-Cohen (2009), Bansal y Roth (2000)).

\subsection{Autoridad institucional}

El primero de los constructos identificados se mueve en el campo de la influencia de los gobiernos sobre todas las organizaciones. La regulación y la presión de los gobiernos para la institucionalización de las organizaciones posiblemente haya sido el factor de más influencia en el inicio o arranque de estos procesos (Bansal y Roth 2000; Brønn y VidaverCohen (2009). Scott (1995) señala a la acción reguladora de los gobiernos como uno de los pilares de las instituciones.

El constructo sugiere que las organizaciones desarrollan sus iniciativas institucionales debido principalmente a las presiones de los gobiernos y los organismos con autoridad y poder para imponer sus decisiones. Aunque la importancia de la coerción en esta acción reguladora ha sido esencial, hoy y cada vez en mayor medida, encontramos que la acción reguladora trata de orientarse hacia otras formas de influencia sobre las organizaciones. De este modo, el cluster que definimos pasa a estar integrado, además de por la inevitable regulación coercitiva, por la autorización normativa que sirve para acreditar el seguimiento de criterios y estándares públicos, por la señalización de las políticas por parte de los gobiernos como un aviso y advertencia para las organizaciones, y por el obligado entendimiento con las administraciones cuando existen fuertes relaciones de negocio o dependencia respecto al sector público.

\subsection{Ventaja en gestión}

Diversas investigaciones (Margolis y Walsh 2003) han puesto de manifiesto que la sociedad afronta con escepticismo los motivos por los que las empresas abordan las iniciativas institucionales pensando que estas actividades se llevan a cabo buscando el interés de la organización (Aguilera et al. 2007). De hecho, en un buen número de estudios, se comprueba que las empresas adaptan sus comportamientos a los esperados por la sociedad para conseguir ventajas económicas (Bansal y Roth 2000; Brønn y Vidaver-Cohen 2009)

Este segundo constructo nos indica que las organizaciones, en muchas ocasiones, desarrollan una cartera de iniciativas institucionales porque los CEOs creen que estas actividades pueden proporcionar una ventaja en gestión, aportando nuevas oportunidades de negocio, o ayudando a la organización a satisfacer las demandas de los propietarios. La ventaja en gestión incluye la participación en las iniciativas gubernamentales mediante la incentivación de actuaciones, la innovación y mejora de los procesos confiando en obtener por ello ventajas competitivas, la creencia de los CEOs respecto a las ventajas para la gestión de la reducción de problemas y costes, el convencimiento de que las iniciativas institucionales conseguirán un mejor acceso a los recursos y la capacidad de captar inversiones, 
el convencimiento de que mediante la integración en redes sociales se conseguirán ventajas de diverso tipo, el prestigio social como una fuente de ventajas a largo plazo y, añadido a lo anterior, la creencia de los CEOs de que las actuaciones filantrópicas aportarán también algún tipo de ventaja gracias a la mejora de la imagen de la organización.

Bansal y Roth (2000), encuentran un constructo similar que definen como 'competitividad' en donde recogen el potencial de las actuaciones que conseguirán mejorar la rentabilidad a largo plazo. Brønn y Vidaver-Cohen (2009), también hallan un constructo en la misma línea al que denominan 'rentabilidad' el cual recoge los motivos de la iniciativa social enfocados a obtener ventajas económicas. Para Scott (1995) lo anterior se encontraría ligado al pilar cognitivo de las instituciones representando lo que racionalmente realizan las corporaciones a partir de los conocimientos y de la interpretación del entorno (Garud et al. 2007).

\subsection{Implicacion social}

La implicación social es el tercer constructo que representa lo que debe hacer una organización responsable. El significado de este constructo comprende todo aquello que se encuentra vinculado al deber ser. Las actuaciones del CEO se realizarán no sólo porque los gobiernos obliguen a realizarlas, o porque dichas actuaciones proporcionen ventajas económicas o financieras, sino porque es el modo correcto de actuar haya o no presiones internas o externas que faciliten o dificulten las iniciativas (Díez-Martín et al. 2010, Glaskiewicz y Colman 2006), teniendo siempre en cuenta los valores y principios sociales y de la propia organización. Scott (1995) destaca como pilar de la institucionalización el aspecto 'normativo' que guía la acción a través de normas de aceptabilidad, la moral y la ética.

De este modo, agrupamos en el constructo aspectos relevantes que los expertos participantes en el estudio podrían, aparentemente, en algún caso, haber incluido en el constructo anterior pero que han identificado no por la ventaja económica que pueden proporcionar sino por el compromiso social que implican. Este matiz conceptual es muy relevante a efecto de establecer el origen de las decisiones institucionales de los CEOs. Encontramos así, la posición ética y los valores corporativos como una cuestión que sin duda debía figurar en el presente constructo pero además, identificamos otro ítem con una gran importancia social como es el mimetismo, iniciativas que se emprenden para sentirse inmerso en el sector y pensar en la organización como una más dentro de un colectivo de organizaciones. Pero entre lo que debe ser, lo que es correcto a la hora de actuar, identificamos también los deseos y aspiraciones de los stakeholders y de los consumidores. Según los participantes en el estudio, se deben satisfacer las demandas de los grupos de presión y los consumidores no por las consecuencias económicas derivadas de la ignorancia de sus requerimientos sino porque eso es lo correcto, porque esa es la responsabilidad de la organización. Por último, también encontramos una cuestión con un significado especial, nos referimos al interés estratégico. La organización, su futuro y su progreso depende de su estrategia y, en consecuencia, dicha estrategia será una garantía para su supervivencia, por lo tanto, si socialmente es significativo que la organización se mantenga y progrese, se deben emprender iniciativas que aseguren su estrategia y evitar la desaparición de la misma.

Por último, la presión para participar en iniciativas sociales a menudo se genera por la propia comunidad corporativa donde los gerentes son presionados por sus compañeros para participar en iniciativas sociales 


\section{CONCLUSIONES}

La perspectiva institucional observa el diseño organizacional, no sólo como un proceso racional, sino más bien como la consecuencia de presiones, aspiraciones, creencias y valores, tanto internas como externas, que hacen que las organizaciones actúen (Hall y Taylor 1996).

Las organizaciones pueden actuar por motivos altruistas, por el interés propio, o por presión gubernamental, e incluso, puede ser que varios motivos se concatenen y actúen juntos como fuerzas desencadenantes de la institucionalización. Nuestro enfoque busca distinguir y separar estas motivaciones sin manifestar mayor aprecio o rechazo por cada una de ellas ya que, por uno u otro motivo, las consecuencias de las actuaciones acaban desembocando en la institucionalización de las organizaciones y eso, por sí mismo, es un factor de progreso.

Un modelo enriquecido de la institucionalización de las organizaciones requiere la consideración de las motivaciones subyacentes. Esta investigación es un intento de avanzar en la comprensión de por qué las empresas son sensibles a la institucionalización. Para ello se propone una tipología que ayudan a describir el origen del proceso corporativo de institucionalización, facilitando la formulación posterior de hipótesis y teorías.

Los resultados de nuestra investigación ayudan a responder ciertas preguntas sobre las motivaciones de los CEO a la hora de participar en iniciativas institucionales. Nuestros resultados refuerzan varios de los temas claves sugeridos en la literatura sobre Teoría Institucional. En particular, encontramos fuerte apoyo para la tipología de motivos que dan origen a las iniciativas institucionales.

Discutimos nuestros resultados sobre los factores desencadenantes de las iniciativas directivas, en el contexto de estos tres temas: Autoridad institucional; Ventaja en gestión; e, Implicación social. Al mismo tiempo, el estudio plantea preguntas nuevas que pueden desafiar las ideas existentes sobre el tema y proporcionar direcciones interesantes para la investigación futura. En particular, nosotros sugerimos que en las organizaciones deben existir factores desencadenantes de las iniciativas institucionales o éstas iniciativas no se producirán. La aparición en mayor o menor medida de motivaciones acaba desarrollando organizaciones distintas, transformándolas gracias al impulso que proporciona el proceso de institucionalización que se genera. Si hay motivación en el CEO, podemos asegurar que antes o después, la organización se transformará en institución y, por tanto, adquirirá legitimidad social. El papel que damos al CEO de una organización, desde un punto de vista práctico, es crucial porque las organizaciones responden a estructuras en las que la jerarquía y la estructura formal es esencial. Nada puede empezar sin el conocimiento y el apoyo del CEO, especialmente cuando se trata de cuestiones que se encuentran en el más alto nivel de la escala de decisión como sucede con los contenidos de los tres constructos identificados más arriba. Transformar una organización tradicional en otra organización institucionalizada requiere no sólo esfuerzo sino sobre todo decisión.

Entre las limitaciones de nuestro trabajo conviene señalar que se circunscribe al aspecto descriptivo de la ciencia. Según Hempel (1965) la ciencia tiene dos funciones básicas, la primera de ellas consiste en describir adecuadamente los objetos que están siendo investigados, la segunda establecer teorías. Las descripciones expuestas en nuestro trabajo pueden ser la materia prima de teorías, pero no son en sí mismas enunciados teóricos. Ini- 
cialmente partimos de una categorización basada en la literatura disponible. Dichas categorías procedían de distintas fuentes pues fueron creadas por estudiosos que enfocaban su interés sobre cuestiones específicas y no sobre la globalidad del tema tratado. Como señala Bacharach (1989), la categorización suele ser especialmente rica y útil como terreno para la construcción de la teoría, sin embargo, entra claramente en el campo de la descripción, no de la teoría.

Posteriormente, dada la variedad y riqueza de las aportaciones previas, decidimos crear una tipología, emulando las ideas de Weber sobre constructo, "Un constructo mental, formado por la síntesis de muchos difusos... fenómenos individuales que son dispuestos, de acuerdo con ciertos puntos de vista, en una constructo analítico unificado" (Bacharach 1989, p. 497). Esta tipología forma parte también del campo de la descripción.

Las limitaciones de esta investigación proporcionan anclajes para futuras investigaciones. Nuestro estudio no analiza el nivel o grado de institucionalización, no se ha establecido una escala al respecto y, por tanto, aunque abre la puerta a su posterior definición, será necesario realizar estudios empíricos para definir el nivel o grado de institucionalización. El modelo no permite a un investigador hacer especulaciones válidas sobre la eficacia relativa de los constructos, aunque sí proporciona información sobre la importancia relativa de las motivaciones, tal como se observa en la Tabla 2, en donde los pesos representan la media del nivel de importancia que otorgaron a cada ítem los participantes.

Tabla 2

\section{Peso de los ítems}

\begin{tabular}{|c|c|c|}
\hline${\text { Ítem } \mathrm{n}^{\circ}}^{\mathrm{C}}$ & Concepto & Peso \\
\hline 6 & Interés estratégico. & 4,3 \\
\hline 8 & Innovación y mejora de procesos. & 4,1 \\
\hline 1 & Regulación coercitiva. & 4,0 \\
\hline 9 & Actitud del consumidor. & 4,0 \\
\hline 12 & Presión de los stakeholders. & 4,0 \\
\hline 14 & Prestigio social. & 3,8 \\
\hline 2 & Autorización normativa. & 3,8 \\
\hline 3 & Incentivación de actuaciones. & 3,8 \\
\hline 7 & Fuertes relaciones con el sector público. & 3,8 \\
\hline 15 & Posición ética y valores corporativos. & 3,7 \\
\hline 10 & Reducción de problemas y costes. & 3,6 \\
\hline 4 & Señalización de políticas públicas. & 3,3 \\
\hline 5 & Acceso a recursos. & 3,3 \\
\hline 13 & Mimetismo. & 2,9 \\
\hline 11 & Integración en redes sociales. & 2,8 \\
\hline 16 & Filantropía. & 2,6 \\
\hline
\end{tabular}


La investigación futura podrá poner a prueba la eficacia y el peso real de las motivaciones, lo que supondrá establecer la relación formal entre las motivaciones que inician e impulsan los procesos de institucionalización y el modo en que estos impulsos iniciales se transforman en actuaciones reales o iniciativas institucionales

\section{REFERENCIAS BIBLIOGRÁFICAS}

Aguilera, R.V.; Rupp, D.E.; Williams, C.A. y Ganapathi, J., 2007. Putting the S back in corporate social responsibility: A multilevel theory of social change in organizations. Academy of Management Review, 32 (3), 836-863.

Anderson L.A.; Gwaltney, M.K.; Sundra, D.L. et al., 2006. Using concept mapping to develop a logic model for the Prevention Research Centers Program. Preventing Chronic Disease. Public health research, practice, and policy, 3 (1), 1-9.

Ashforth, B.E. y Gibbs, B.W., 1990. The double-edge of organizational legitimation. Organization Science, 1 (2), 177-194.

Bacharach, S.B., 1989. Organizational theories: Some criteria for evaluation. Academy of Management Review, 14 (4), 496-515.

Bansal, P. y Roth, K., 2000. Why companies go green: A model of ecological responsiveness. Academy of Management Journal, 43 (4), 717-736.

Barnett, M.L., 2007. Tarred and untarred by the same brush: exploring interdependence in the volatility of stock returns. Corporate Reputation Review, 10 (1), 3-21.

Barth, M.C., 2004. A low-cost, post hoc method to rate overall site quality in a multisite demonstration. American Journal of Evaluation, 25 (1), 79-97.

Bartlett, A. y Preston, D., 2000. Can ethical behaviour really exist in business? Techniques, 23 (2), 199-209.

Baum, J. y Oliver, C., 1991. Institutional linkages and organizational mortality. Administrative Science Quarterly, 36 (2), 187-218.

Brønn, P.S. y Vidaver-Cohen, D., 2009. Corporate motives for social initiative: legitimacy, sustainability, or the bottom line? Journal of Business Ethics, 87, 91-109.

Bitektine, A., 2011. Toward a theory of social judgments of organizations: the case of legitimacy, reputation, and status. Academy of Management Review, 36 (1), 151-179.

Campbell, J.L., 2007. Why would corporations behave in socially responsible ways? An institutional theory of corporate social responsibility. Academy of Management Review, 32 (3), 946-967.

Carroll, A.B., 1979. A three-dimensional conceptual model of corporate performance. Academy of Management Review, 4 (4), 497-505.

Chen, J.C., Patten, D.M. y Roberts, R.W., 2008. Corporate charitable contributions: A corporate social performance or legitimacy strategy? Journal of Business Ethics, 82, 131-144.

Cho, C.H. y Patten, D.M., 2007. The role of environmental disclosures as tools of legitimacy: A research note. Accounting, Organizations and Society, 32 (7-8), 639-647.

Cruz-Suárez, A.; Prado-Román, C. y Díez-Martín, F., 2014. Por qué se institucionalizan las organizaciones. Revista Europea de Dirección y Economía de la Empresa 23 (1), 22-30.

Cyert, R.M. y March, J.G., 1963. A behavioral theory of the firm, Englewood Cliffs, NJ: Prentice-Hall. 
Dacin, M.T.: Oliver, C. y Roy, J.P., 2007. The legitimacy of strategic alliances : an institutional perspective. Strategic Management Journal, 28 (2), 169-187.

Davis, K., 1973. The case for and against business assumption of social responsibilities. Academy of Management Journal, 16 (2), 312-322.

Deephouse, D.L., 1996. Does isomorphism legitimate? Academy of Management Journal, 39 (4), 1024-1039.

Díez-Martín, F.; Medrano García, M.L. y Díez de Castro E.P. 2008. Los grupos de interés y la presión medioambiental. Cuadernos de Gestión, 8 (2), 81-96

Díez-Martín, F.; Blanco-González, A., y Prado-Román, C. 2010. Medición de la legitimidad organizativa: el caso de las Sociedades de Garantía Recíproca. Cuadernos de Economía y Dirección de la Empresa, 43, 115-144.

Díez-Martín, F.; Prado-Román, C. y Blanco-González, A., 2013. Efecto del plazo de ejecución estratégica sobre la obtención de legitimidad organizativa. Investigaciones Europeas de Dirección y Economía de la Empresa, 19 (2), 120-125.

DiMaggio, P.J. y Powell, W.W., 1983. The iron cage revisited: institutional isomorphism and collective rationality in organizational fields. American Sociological Review, 48 (2), 147-160.

Dornbusch, S.M. y Scott, W.R., 1975. Evaluation and the exercise of authority. San Francisco: Jossey-Bass.

Drucker, P., 1999. Management challenges for the 21st century. Oxford: Butterworth Heinemann.

Easton, G.S. y Jarrell, S.L., 1998. The effects of total quality management on corporate performance: An empirical investigation. The Journal of Business, 71 (2), 253-307.

Fine, A. H., Thayer, C. E., y Coghlan, A. T. (2000). Program evaluation practice in the nonprofit sector. Nonprofit Management and Leadership, 10(3), 331-339.

Freeman, R.E., 1984. Strategic management: A stakeholder approach. Boston: Pitman.

Gardberg, N.A. y Fombrun, C.J., 2006. Corporate Citizenship: Creating Intangible Assets Across Institutional Environments. Academy of Management Review, 31 (2), 329-346.

Garud, R.; Hardy, C. y Maguire, S, 2007. Institutional Entrepreneurship as Embedded Agency: An Introduction to the Special Issue. Organization Studies, 28 (7), 957-969.

Glaskiewicz, J. y Colman, M.S., 2006. Collaboration between corporations and nonprofit organizations. En: Powell, W. W. y Steinberg, R. Eds. The nonprofit sector: A research handbook. New Haven CT: Yale University Press, 180-205.

Graafland, J. y Van der Ven, B., 2006. Strategic and moral motivation for corporate social responsibility, Journal of Corporate Citizenship, 22, 111-124.

Green Jr.; S.E., Li, Y. y Nohria, N., 2009. Suspended in self-spun webs of significance: A rhetorical model of institutionalization and institutionally embedded agency. Academy of Management Journal, 52 (1), 11-36.

Greenwood, R. y Suddaby, R., 2006. Institutional entrepreneurship in mature fields: The big five accounting firms. Academy of Management Journal, 49 (1), $27-48$.

Hahn, T. y Scheermesser, M., 2006. Approaches to corporate sustainability among german companies. Corporate Social Responsibility and Environmental Management, 13 (3), $150-165$.

Hall P. A, y Taylor, R. C. R.., 1996. Political science and the three new institutionalisms. Political. Studies. 44, 936-957. 
Hambrick, D.C. y Mason, P.A., 1984. Upper echelons: The organization as a reflection of its top managers. Academy of Management Review, 9 (2), 193-206.

Hart, S.L., 1995. A natural-resource-based view of the firm. Academy of Management Review, 20 (4), 986-1014.

Hatchuel, A., 2001. Towards design theory and expandable rationality: The unfinished program of Herbert Simon. Journal of Management and Governance, 5 (3-4), 260-273.

Hempel, C., 1965. Aspects of scientific explanation. New York: Free Press.

Heugens, P. y Lander, M.W., 2009. Structure! Agency! (and other quarrels): a meta-analysis of institutional theories of organization. Academy of Management Journal, 52 (1), 61-85.

Huebner, T. A. (2000). Theory-based evaluation: Gaining as shared understanding between school staff and evaluation. En Rogers, P. J.; Hacsi, T. A.; Petrosino, A. y Huebner, T.A., eds., Program theory in evaluation: Challenges and opportunities. New directions for evaluation, Vol. 87. San Francisco: Jossey-Bass.

Ingram, P. y Silverman, B. S. 2002. The new institutionalism in strategic management. Advances in Strategic Management, 19, 1-32.

Isaacson, W., 2011. Steve Jobs. Barcelona: Random House Mondadori.

Jackson, K. M. y Trochim, W. M. K., 2002. Concept mapping as an alternative approach for the analysis of open-ended survey responses. Organizational Research Methods, 5 (4), 307-336.

Kane, M. y Trochim W.M.K., 2007. Concept Mapping for Planning and Evaluation. Thousand Oaks: Sage.

Kozinets, R.V. y Handelman, J.M., 2004. Adversaries of consumption: consumer movements, activism, and ideology. Journal of Consumer Research, 31 (3), 691-704.

Kruskal, J.B. y Wish, M.W., 1978. Multidimensional Scaling, 11, Newbury Park. California: Sage University Paper.

Maguire, S. y Hardy, C., 2009. Discourse and deinstitutionalization : The decline of DDT. Academy of Management Journal, 52 (1), 148-178.

March, J. y Simon, H.A., 1958. Organizations, New York: Wiley.

Margolis, J.D. y Walsh, J.P., 2003. Misery loves companies: Rethinking social initiatives by business. Administrative Science Quarterly, 48 (2), 268-305.

Matten, D. y Moon, J., 2008. "Implicit" and "Explicit" CSR: A conceptual framework for a comparative understanding of corporate social responsibility. Academy of Management Review, 33 (2), 404-424.

Meyer, J. W., \& Rowan, B. 1983. The structure of educational organizations. In J. W. Meyer \& W. R. Scott (Eds.), Organizational environments: Ritual and rationality, 71-97. Beverly Hills, CA: Sage.

Ostrom, E., 2000. Collective action and the evolution of social norms. Journal of Economic Perspectives, 14(3), 137-158.

Pache, A.C. y Santos, F., 2010. When worlds collide: The internal dynamics of organizational responses to conflicting institutional demands. Academy of Management Review, 35 (3), 455-476.

Palmer, D.; Biggart, N. y Dick, B., 2008. Is the new institutionalism a theory? En Greenwood, R.; Oliver, C.; Suddaby, R. y Sahlin, K., eds. The Sage handbook of organizational institutionalism. London: Sage Publications, 739-768. 
Peloza, J., 2003. Using corporate social responsibility as insurance for financial performance. California Management Review, 48 (2), 52-73.

Pfarrer, M.D.; DeCelles, K.A.; Smith, K.G. y Taylor, M.S., 2008. After the fall: Reintegrating the corrupt organization. Academy of Management Review, 33 (3), 730-749.

Porter, M.E. y Van der Linde, C., 1995. Green and competitive : Ending the stalemate green and competitive. Harvard Business Review, 73 (5), 120-134.

Rehbein, K.; Waddock, S. y Graves, S.B., 2004. Understanding shareholder activism: Which corporations are targeted? Business Society, 43 (3), 239-267.

Riquel, F.J. y Vargas, A., 2013. Las presiones institucionales del entorno medioambiental: aplicación a los campos de golf. Revista Europea de Dirección y Economía de la Empresa, 1 (1), 29-38.

Roberts, P.W. y Greenwood, R., 1997. Integrating transaction cost and institutional theories: Toward a constrained-efficiency framework for understanding organizational design adoption. Academy of Management Review, 22 (2), 346-373.

Rosas, S. R., 2005. Concept mapping as a technique for program theory development: An illustration using family support programs. American Journal of Evaluation, 26(3), 389-407

Rosas, S.R. y Camphausen, L.C., 2007. The use of concept mapping for scale development and validation in evaluation. Evaluation and Program Planning, 30, 125-135.

Ruef, M. y Scott, W.R., 1998. A multidimensional model of organizational legitimacy: Hospital survival in changing institutional environments. Administrative Science Quarterly, 43 (4), 877-904.

Russo, M. V y Fouts, P.A., 1997. A resource-based perspective on corporate environmental performance and profitability. Academy of Management Journal, 40 (3), 534-559.

Scott, W.R., 1987. The adolescence of institutional theory. Administrative Science Quarterly, 32 (4), 493-511.

Scott, W.R., 1995. Institutions and organization. Thousand Oaks: Sage Publications.

Selznick, P., 1957. Leadership in administration: A sociological interpretation. New York: Harper and Row.

Selznick, Philip 1996. Institutionalism "Old" and "New". Administrative Science Quarterly, 41 (2), 270-277.

Sen, S. y Bhattacharya, C.B., 2001. Does doing good always lead to doing better? Consumer reactions to corporate social responsibility. Journal of Marketing Research, 38(2), 225-243.

Sharma, S., 1996. Applied Multivariate Techniques. New York: John Wiley \& Sons.

Shrivastava, P., 1995. The role of corporations in achieving ecological sustainability. Academy of Management Review, 20 (4), 936-960.

Sine, W.D.; David, R.J. y Mitsuhashi, H., 2007. From plan to plant: Effects of certification on operational start-up in the emergent independent power sector. Organization Science, 18 (4), 578-594

Singh, J. V.; Tucker, D.J. y House, R.J., 1986. Organizational legitimacy and the liability of newness. Administrative Science Quarterly, 31 (2), 171-193.

Spence, M., 1973. Job market signaling. Quarterly Journal of Economics, 87 (3), 355-374.

Starr, J.A. y MacMillan, I.C., 1990. Resource cooptation via social contracting: Resource acquisition strategies for new ventures. Strategic Management Journal, 11 (4), 79-92. 
Steurer, R. et al., 2005. Corporations, stakeholders and sustainable development I: A theoretical exploration of business-society relations. Journal of Business Ethics, 61 (3), 263-281.

Suchman, M.C., 1995. Managing legitimacy: Strategic and institutional approaches. Academy of Management Review, 20 (3), 571-610.

Terlaak, A., 2007. Order without law? The role of certified management standards in shaping socially desired firm behaviors. Academy of Management Review, 32(3), 968-985.

Trochim, W.M.K., 1989. Concept Mapping: Soft science or hard art? Evaluation and Program Planning, 12 (1), 87-110.

Trochim, W., 1993.The Reliability of Concept Mapping. meyer, Dallas, Texas, November 6 .

Tudway, R. y Pascal, A.M., 2006. Corporate governance, shareholder value and societal expectations. Corporate Governance, 6 (3), 305-316.

Vaara, E. y Tienari, J., 2008. Note. A discursive perspective on legitimation strategies in multinational corporations. Academy of Management Review, 33 (4), 985-993.

Williams, R.J. y Barrett, J.D., 2000. Corporate philanthropy, criminal activity, and firm reputation: Is there a link? Journal of Business Ethics, 26 (4), 341-350.

Williamson O. E., 1991. Comparative Economic Organization: The Analysis of Discrete Structural Alternatives. Administrative Science Quarterly, 36 (2), 269-296.

Williamson, O. E., 2000. The New Institutional Economics: Taking Stock, Looking Ahead. Journal of Economic Literature, 38 (3), 595-613.

Yampolskaya, S.; Nesman, T. M.; Hernandez, M. and Koch, D., 2004. Using Concept Mapping to Develop a Logic Model and Articulate a Program Theory: A Case Example. American Journal of Evaluation, 25 (2), 191-207.

Zimmerman, M.A. y Zeitz, G.J., 2002. Beyond survival: Achieving new venture growth by building legitimacy. Academy of Management Review, 27 (3), 414-431.

\section{APÉNDICE}

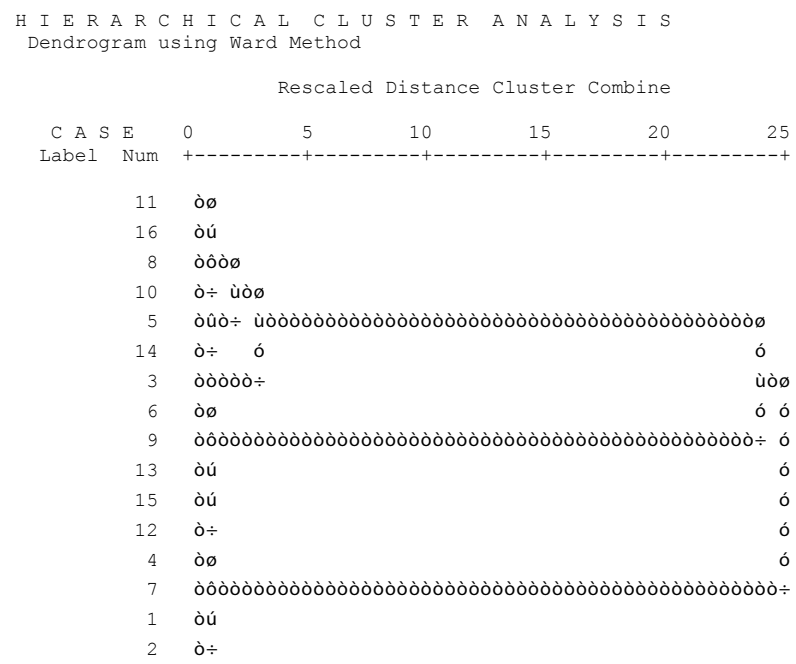

